\title{
Informe bibliográfico sobre Schelling
}

\section{Bibliographic report on Schelling}

\author{
ALEJANDRO ROJAS JIMÉNEZ \\ Ludwig-Maximilians-Universität München (Alemania)
}

Recibido: 02-08-2011 Aprobado definitivamente: 29-08-2011

\begin{abstract}
RESUMEN
Buscando atraer al lector a la bibliografía aquí informada, he procedido en primer lugar a presentar la obra de Schelling haciendo hincapié en el papel que juega para entender el quehacer filosófico reciente, incluso aquel aparentemente más alejado. Dicha presentación es seguida de una indicación de las ediciones de sus obras completas, un registro de sus obras ordenadas por año, y una selección de la bibliografía secundaria con especial atención a las contribuciones del grupo de investigación sobre Schelling de la Universidad de Málaga, cuya labor es rectora en este análisis de la influencia de Schelling en la filosofía reciente.
\end{abstract}

PALABRAS CLAVES

DIFERENCIA ONTOLÓGICA, ABSOLUTE PRIUS, LOS LÍMITES DEL CONCEPTO, EL TEMA DE LA FILOSOFÍA, BIBLIOGRAFÍA

\begin{abstract}
In an attempt to seek to attract the reader to the bibliography reported here, I proceed first to present Schelling's work emphasizing the importance of his role to understand the most recent philosophy, and even more seemingly distant philosophy. This presentation is followed by an index of Schelling's complete works editions, a list of Schelling's works organized by year, and a selection of secondary literature on Schelling, with special attention to the contribution of the research group on Schelling at the University of Malaga, whose work is first-line in the analysis of Schelling's influence on recent philosophy.
\end{abstract}

CC Contrastes. Revista Internacional de Filosofía, vol. XVII (2012), pp. 343-366. ISSN: 1136-4076

Departamento de Filosofía, Universidad de Málaga, Facultad de Filosofía y Letras

Campus de Teatinos, E-29071 Málaga (España) 
KEY WORDS

ONTOLOGICAL DIFFERENCE, ABSOLUTE PRIUS, THE LIMITS OF THE CONCEPT, THE SUBJECT OF PHILOSOPHY, BIBLIOGRAPHY

\section{Presentación de la obra de Schelling}

La FiLOSOfía DE Schelling va adquiriendo poco a poco el protagonismo que le corresponde. A la sombra de Hegel y de su sistema absoluto, el filósofo de Leonberg y su defensa de que una identidad absoluta de corte hegeliano no sólo no es posible sino indeseable, sólo en los últimos tiempos va atrayendo los oyentes merecidos, en parte gracias a Heidegger y su atractiva aseveración de que Schelling es la cumbre de la metafísica del idealismo alemán. Los oyentes son sin embargo aún escasos, y Schelling sigue a la sombra del idealismo absoluto de Hegel ¿Dónde se encuentra la razón de esta desatención recurrente? Y no menos pertinente ¿En qué se basa el juicio de que esta desatención es injusta o desmerecida?

El interés de su estudio podría reivindicarse haciendo referencia por ejemplo a su influencia relevante en autores como Kierkegaard, Bakunin o Engels, que asistieron a sus clases, y que aunque no quedaron del todo satisfechos, recibieron el espíritu de una filosofía que después cada uno desarrollaría a su modo. Sin embargo, la contribución a la historia de la filosofía de Schelling es mucho mayor que una mera influencia en el espíritu anti-hegeliano de la época. Es mi intención a continuación, y como modo de presentación de la obra de Schelling, introducir, en esta breve presentación de su obra, una consideración poco recurrida para justificar la promoción del interés hacia Schelling: el papel fundamental que éste juega para entender el quehacer filosófico actual.

Ante una afirmación semejante gran parte del abanico de filósofos actuales podrían escandalizarse y alegar, por ejemplo, que la filosofía de la ciencia, de la mente o la política, entre otras, poco tienen que ver con aquel ejercicio especulativo idealista ejercido por el filósofo de Leonberg. No parece, en efecto, que este tipo de filosofías tengan nada que deberle a Schelling, y mucho menos en lo tocante al modo en el que ellos despliegan el quehacer filosófico. Más mi afirmación no ha descuidado la existencia de este tipo de filosofías contemporáneas que tanto difieren respecto del modo de hacer filosofía de Schelling. La tesis propuesta bien podría enunciarse así: Schelling es el punto central, o al menos un punto decisivo, para entender la situación actual de la filosofía, desvertebrada y desunida, y ello aún cuando parezca evidente que en muchas de sus líneas de investigación el quehacer filosófico actual no tiene que ver con el ejercido por el propio Schelling. Schelling no es considerado en esta tesis 
como un origen a partir del cual se desarrollen esas filosofías «de», sino que Schelling es concebido en este caso como un punto de efervescencia para una suerte de resurgimiento de un modo de entender el quehacer filosófico que se aleja de lo hecho por él mismo.

De entrada, el tema que le preocupa a Schelling está bastante alejado de los intereses de estos quehaceres filosóficos. Su tema es la automanifestación de Dios. Lo cual propondré entender como sigue: la filosofía de Schelling es una reflexión acerca del origen del ser del mundo; Schelling piensa este origen como un crear (Zeugen) en el que lo incondicional se da su propia medida en un proceso de Kunstproduktion en el que la fuerza productiva (Produktionskraft) se expresa y se oculta en los productos dado que la incondicional productividad no es inmediatamente manifestable. Lo incondicional es así la libertad pensada como Kant la pensó, como autolegislación (Selbstgesetzbung), aunque obviamente la filosofía de Schelling se separa de Kant al pensar la productividad como construirende Thätigkeit que es la unidad de productividad y producto. Dice H. Krings: "Naturaleza y sujeto quieren decir pura productividad, la cual representa en cada uno de sus productos eso mismo que sin embargo es diferente a ellos, a saber, la actividad absoluta". Aparentemente poco interés puede tener esta filosofía para los filósofos que hacen filosofía «de». Si bien, tampoco levanta pasiones entre los amigos del quehacer especulativo tradicional y originario, en tanto que Schelling hace toparse al idealismo (y en general a la filosofía) con algo que no puede ser conceptualizado, lo schlechterdings trasscendente Seyn o lo absolute Prius que ningún concepto ni idea puede abarcar; que sólo se conoce por sus consecuencias, o mejor, «en» sus consecuencias: la sustancia incondicional considerada en sus consecuencias según la fórmula $\mathrm{A} / \mathrm{a}$, donde A/a es una consecuencia de A al tiempo que una sustancia particular propia que actúa libremente por sí misma. ¿Pero y A en tanto que A? ¿Es algo de lo que no se puede habar? ¿Algo de lo que mejor es callarse? Schelling buscó el modo de hablar acerca de lo inefable, y no pudo crear algo sólido. Así, en medio de dos líneas enfrentadas (los que se acercan más al neopositivismo y las filosofías prácticas, y los que se acercan más a la especulación teórica), y sin ser capaz de crear algo sólido, su filosofía corría la suerte que le espera a los olvidados, hasta que Heidegger reparó en una peculiaridad decisiva de su sistema que lo convertían en el filósofo «irrenunciable» para entender todo un periodo de la historia, precisamente esa historia reciente de la filosofía, que tiene como una

1 H. Krings, «Narur als Subjekt. Ein Grundzug der spekulativen Physik Schellings», en: Heckmann-Krings-Meyer (eds.), Natur und Subjektivität. Zur Auseiandersetzung mit der Naturphilosophie des jungen Schelling. Referate, voten und Protokolle der II. Internationalen Schellings-Tagung Zürich 1983, Stuttgart-Cannstatt: frommann-holzboog, 1985, p 123.

2 Cfr., PhU, SW VII, p. 344. 
de sus características definitorias más típicas su incapacidad para ponerse de acuerdo en la consideración de en qué pueda consistir el quehacer filosófico mismo.

Heidegger dirá en el seminario de 1941 que Schelling lo que está pensando es el ser que es sólo ser, y que por ser sólo ser no «es» en el mismo sentido que los demás seres, sino un ser incondicional que precisamente es el origen de lo siendo. ${ }^{3}$ Heidegger emplea la expresión «west» para sustituir a «ist», en referencia a que ese ser es Wesen y no Sein: la sustancia incondicional que desde un punto de vista es sólo ser, pero desde otro punto de vista es siempre «en» sus consecuencias. Esta diferencia ontológica, que no habla de seres distintos, sino de un ser considerado desde puntos de vistas distintos, es el gran pilar sobre cuya reformulación se va a levantar el edificio de la filosofía tardía de Heidegger, pero es el pilar a su vez de la obra de Schelling, si bien, también en Schelling hay una reformulación bien interesante cuando la distinción primigenia entre Wesen y Form se ve sometida a un proceso de reformulación que acaba por desembocar en la distinción entre Wirklichkeit y Möglichkeit, esto es, cuando Schelling se percata de que no se trata de pensar la causa y sus consecuencias (la doctrina de las potencias, eje de su filosofía anterior), sino la causa de las causas, de tal modo que la doctrina de las potencias vendría a encajar en el marco de las Möglichkeiten, al tiempo que se introduciría una cuarta causa como causa de las causas: das absolute Einzelwesen que es aition tou einai, la realidad (Wirklichkeit) del ser de aquellas posibilidades, la cual quedaría fuera del proceso cósmico, siendo sólo oteada de mal modo en las oscuridades de un abismo donde la razón difícilmente encuentra suelo y que Schelling creyó que sólo de un modo pseudomístico, mitológico o simbólico podía ser considerada. La expresión usada en 1809, Ungrund, ha sido sin duda una expresión con mucho éxito. Se trata de una formulación paralela a la noción de Indifferenz, porque ese $U n$ es el prefijo alemán para el in latino. La idea es que fundamento parece designar dos cosas: lo que funda y lo fundado; Schelling sin embargo piensa un ser que precede a toda oposición, ${ }^{4}$ un ser que es Eins von Allem ¿Mas puede hablarse acaso de este ser de algún modo? ¿Y por qué iba a ser este asunto tan crucial para entender el destino de la filosofía?

En principio no parece que ni el tema ni el método del filosofar de Schelling puedan dar pie a la tesis general aquí defendida de que en Schelling se encuentra la clave para entender la situación de la filosofía actual. La clave de

3 «Das Seyn als dasjenige west, was allein ist». Heidegger, M., «Ausgewählte Stücke aus den manuskripten zur Vorbereitung der Schelling-Seminars S.S. 1941», in: Heidegger, Schelling Abhandlung über das Wesen deer menschlischen Freiheit (1809), Tübingen: Niemayer, 1971, p. 231.

4 PhU, SW VII, p. 406 
la cuestión para entender dicha afirmación tiene que ver con eso encontrado que bien parece que nos obliga a callar. Gran parte de la filosofía que actualmente se hace, parte de una suerte de proclamación del silencio ante estos temas una vez que se ha perdido la confianza en que se pueda hablar de ellos. Y gran parte de la culpa de esta falta de confianza tiene que ver con el estado de la cuestión allí donde lo lleva Schelling frente a Hegel. En este sentido acusa Falgueras a Schelling de haber llevado la filosofía a la «perplejidad». ${ }^{5}$ Como un intento de superar este estado de perplejidad, al otro lado del panorama filosófico se mantiene el esfuerzo por no renunciar a estas cuestiones que tradicionalmente habían sido la señal de identidad de esta ciencia primera. Sea como sea, se hace filosofía contra Schelling. Y es así como quiero presentarles ese papel, a mi juicio fundamental, que Schelling juega en la explicación del panorama filosófico actual desvertebrado y desunido. Se trata pues de un «a partir» de Schelling bajo la forma de un «ir en contra» de Schelling, en función de ese lugar al que Schelling hace arribar a la filosofía: «un prius absoluto que no puede ni ha de ser demostrado y que sólo se nos da a conocer a posteriori a través de sus manifestaciones libres».

En este ir en contra de Schelling, hemos seleccionado al menos dos grandes «bandos». Por un lado los que proclaman un silencio que se reivindica partiendo del desconocimiento del sentido de un callar semejante, amparándose en una suerte de estrategia gorgiana del tipo: si existiera no podría ser conocido, y si pudiera ser conocido no podría ser expresado. No mejores amigos encuentra sin embargo Schelling entre los filósofos tradicionales, que esquivan las graves acusaciones de Schelling acerca de la filosofía negativa, y se apresuran a hablar de lo que Schelling avisa que es inefable. Estos también se ven obligados a ir también contra Schelling: contra su mitología y su simbolismo, si quieren demostrar que de hecho es posible designar y hablar de eso ante lo que Schelling considera que toda idea sucumbe. Y así las cosas, Schelling está en medio de una contienda que él mismo ha provocado. Por su parte, los amigos del filosofar de Schelling, caso del segundo Heidegger, corren la misma suerte que Schelling, situados en el centro de las críticas de ambos lados del panorama filosófico.

5 «La noción de un prius absoluto que no puede ni ha de ser demostrado y que sólo se nos da a conocer a posteriori a través de sus manifestaciones libres, cuando es entendida como respuesta a aquellos interrogantes fundamentales, viene a equivaler a un "porque sî" o, lo que es igual, a ningún porqué. Las expectativas de un porqué fundamental que dotara de sentido a la razón quedan insatisfechas, y la razón permanece sumida en la perplejidad» Falgueras, I, «Del saber absoluto a la perplejidad. La evolución de la doctrina de las potencias en Schelling», en: Revista de filosofía 6 (1983), p. 33 . 
Schelling es sin duda un envite para ambas posiciones, un punto de encuentro en el que confluye el origen de las filosofías que se hacen tras el idealismo, pero un origen que desborda a muchos, y ante el cual se retrocede. Se busca en Nietzsche, mucho más asequible y claro que el idealista, el origen del fin del modo de hacer filosofía tradicional. La filosofía de Nietzsche y su «rebeldía» se lleva a cabo sobre un terreno ya preparado, que él se limita a anunciar como un «profeta» que en lugar de comunicar lo que vendrá avisa acerca de lo que ya pasó. Bien es cierto que a Schelling no se le pueden atribuir frases del estilo «Dios ha muerto» ${ }^{6}$, pero basta observar las críticas de ateísmo que recibió Schelling para percatarse de que también Schelling, a su modo, había impulsado cierto ataque contra el Dios de cierta filosofía. No es Nietzsche el inicio del fin de la filosofía moderna como se enseña en los institutos de secundaria donde Schelling difícilmente encontraría oyentes preparados, sino el idealista de Leonberg, al lado del cual Nietzsche es, me atreveré a afirmar, sólo un apéndice que en ningún caso alcanza la hondura y vitalidad del pensamiento schellingiano. Las grandes intuiciones sobre las que se levantan las nociones de voluntad de poder y eterno retorno, o la dualidad Apolo-Dionisio, estaban ya actuando en la filosofía de Schelling de forma manifiesta. Así, basta leer las breves páginas del escrito sobre la libertad para encontrar allí la distinción entre las fuerzas vivas y enfrentadas que define como voluntad universal y voluntad particular, y también la consideración de que el ser es voluntad, lo que le lleva a pensar una actividad incesante que Leyte ha caracterizado como Kontinuität ${ }^{7}$ en tanto que designa el proceso de comparecencia de un absoluto que comparece siempre bajo distintas figuras en una sucesión que no encuentra fin, pero donde observamos una clara finalidad (télos): la manifestación del ser absoluto en cada determinación.

Dicho lo cual, quiero dejar patente que no considero que sea necesario entender de dónde viene el actual recelo hacia la teoría pura. Evidentemente no es necesario, ni en la mayoría de los casos se gana nada, exhortando al estudio de Schelling para entender el actual recelo a la teoría y especulación. La lectura de Schelling, y mi llamamiento a que se analice prometiendo que se encontrará en ella la razón de la situación de desorientación actual del quehacer filosófico, es una lectura que tiene que ser sencillamente voluntaria y libre, fruto de la curiosidad científica, y en cualquier caso, estando avisados de que

6 AsZ, Stuttgart: Kröner, 1988, p. 96.

7 «Kontinuität ist jedoch der beste Name, um diesem Signifikanten der Zeit zu bescreiben, in den sich die Dinge einzuschreiben scheinen. Und außerdem begründet diese Kontinuität auch den Vorgang, um die Differenz transzendental/empirisch zu tilgen, die sich gerade ausgehend von dieser Kontinuität auflöst.» Leyte, «Zeit-Denken - Zu einen nicht begrifflichen Zugang zur Zeit bei Schelling und Heidegger», en Hühn-Jatzen (Eds.), Heideggers Schelling-Seminar (1927/28), Stuttgart-Bad Canstatt: frommannn-holzboog, 2010, p. 152. 
al final de la aventura que supone sumergirse en la obra de Schelling, no se alcanza ninguna luz para atacar al nuevo positivismo ni tampoco una postura en la que la teoría especulativa salga malparada. A Schelling no se debe ir en busca de respuestas, sino en busca del problema que él no resuelve y que acaba con el idealismo aunque su filosofía sea de pleno derecho idealista. Después de Schelling son muchos quienes desisten de buscar una solución al problema que aquel no sabe resolver colocándose en el inicio de otro tipo de filosofía. Y no sólo el quehacer filosófico tradicional, ni el nuevo positivismo, sino que todas esas corrientes filosóficas post-idealistas como la fenomenología, el existencialismo o la hermenéutica son una respuesta al punto de llegada, al límite, al que nos ha arribado el pensamiento del de Leonberg. Estamos habituados a entenderlas en relación a Hegel, no sólo porque Hegel es más conocido, sino porque el propio Schelling supone una suerte de ajuste de cuentas con Hegel, el primer gran antihegeliano. El asunto está en que en este ajuste de cuentas, el lugar alcanzado es una bomba de relojería, porque Schelling es el filósofo del empirismo metafísico, de la especulación positiva, el punto de fusión de elementos que al fusionarse sólo pueden explotar y dividir la filosofía en líneas irreconciliables. Y así, en tales circunstancias, su lectura es al mismo tiempo tan fundamental para hacerse cargo de la actual situación de la filosofía, como problemática y escurridiza; y su punto de llegada un punto de partida para algo totalmente distinto.

Debe decirse antes de nada, que estoy presuponiendo que existe algo así como «la» filosofía de Schelling. Parecería una presunción legítima. Pero es tal la perplejidad en la que la lectura de Schelling nos somete, que incluso los especialistas más reconocidos parecen haber renunciado a afirmar siquiera tal cosa. De hecho, una de las acusaciones que se le hacen a Heidegger es no haber sabido distinguir etapas en su pensamiento. Etapas que para muchos, y hablamos de filósofos de renombre como T. Buchheim, son inconmensurables entre sí. No se trata de una posición aislada. Me temo que lo habitual es que la obra de Schelling se considere como una obra dispersa, fragmentada, donde no cabe encontrarse con «una» filosofía, sino con una pluralidad de desarrollos distintos que hacen imposible hablar de una unidad. Tilliete estaría a la cabeza de los defensores de esta consideración que hacen de la filosofía de Schelling une philosophie en devenir. Jacobs, en la Schelling-Kommission de la Academia Bávara de las Ciencias, y Leyte en España, podrían representar bien la otra línea quizás menos difundida, pero sólida y valiente, que se atreve a afirmar constancias (aunque con prudencia) allí donde otros sólo ven diferencias insalvables; y ello en virtud de su capacidad para hacerse con el sentido profundo de las ideas, yendo más allá de las expresiones circunstanciales de las distintas etapas de su pensamiento. 
Me limitaré a comentar brevemente la posición de Jacobs, junto a quien he tenido la posibilidad de trabajar durante mi estancia de investigación financiada por la Humboldt-Stiftung. Jacobs considera que la mejor manera de despertar la curiosidad científica que atraiga el interés de los filósofos hacia Schelling, es precisamente señalando cuál es la idea de fondo que se va reformulando constantemente en su devenir especulativo. ${ }^{8}$ Expondré brevemente esta idea poniéndola en relación con la defensa de la filosofía de Schelling que hace Heidegger y que ha sido anunciada a lo largo de este comentario. De entrada debe ser comentada una notable diferencia. El propio Heidegger encabeza a un grupo cada vez más numeroso que se acerca a Schelling atraído por un aspecto bastante particular y concreto de la filosofía de Schelling que, como en el caso de Heidegger, normalmente se reduce al famoso escrito sobre la libertad. Lo cual se aleja de la intención de Jacobs. Si bien, si Heidegger no distingue épocas es, seguramente, porque sólo conoce bien una parte concreta de la obra de Schelling; y sólo esa le ha interesado. Y sin embargo, aun partiendo de esta enorme desemejanza, la tesis de Jacobs, y su búsqueda de aquella idea que vertebre el conjunto, coincidirían con la propuesta de Heidegger en lo fundamental, porque lo que está en el conjunto también lo está en las partes, de modo que el trabajo minucioso de Jacobs de buscar esa idea de fondo en las distintas etapas, también puede ser visto por Heidegger en esa etapa concreta que a él sólo le interesó.

Heidegger, que considera que las ideas de voluntad de poder y eterno retorno son expresiones de la diferencia ontológica de la filosofía de Schelling, había resumido en 1936 la filosofía de Schelling como sigue: una defensa de la voluntad como ser último de las cosas o ser originario (esencia), que se desarrolla como una defensa de la libertad entendida básicamente como esa capacidad para insistir en la productividad (existencia), y por consiguiente, en una oposición confesa al sistema absoluto hegeliano que se opondría a aquellos dos pilares básicos mencionados, en la medida que éste busca la identificación de ser y existencia en una suerte de transformación de la sustancia en sujeto que supondría a la postre el fin -en el sentido de cese- de aquella productividad originaria y libre. Esta idea, como ha sido dicho con anterioridad, el propio Heidegger la resume diciendo que lo que está pensando Schelling es el ser que es sólo ser, y que -por ser sólo ser- no «es» en el mismo sentido que los demás seres, sino un ser incondicional que precisamente es el origen o fundamento de toda otra modalidad del ser mediante el cual se manifiesta. Creo sin embargo, que Heidegger utiliza esta idea para desarrollar una filosofía propia en la medida en que Heidegger recupera este «modelo» para pensar a su vez la relación

8 Jacobs, W.G., Schelling lesen, Suttgart-Cannstatt: frommann-holzboog, 2004. 
de identidad entre el Seinsgeschick y las distintas figuras del fundamento que acontecen en la historia. ${ }^{9}$

Jacobs por su parte, se pliega más a la obra de Schelling y enuncia así el hilo vertebrador del sistema: «el Dios libre, como lo contrapone Schelling, debe ser pensado como anfangen könnend. No es sólo que él pueda iniciar algo, sino que él mismo tiene que poder iniciar, o dicho de otra manera: él tiene que querer ser Dios mismo. Las construcciones que Schelling necesita para desplegar este pensamiento, esto es, este proceso teogónico, no podemos aquí exponerlas. Basta extraer la conclusión de que Dios tiene una historia.» ${ }^{10}$ enunciada con otras palabras esta tesis dice así: la identidad de la actividad incondicional del ser en tanto que real (Wirklichkeit entendida en el fondo como actividad) y el producto condicionado en tanto que posibilidad a través de la cual aquella fuerza real se manifiesta, no puede ser pensada en términos de identidad absoluta, sino como una unidad donde la identidad absoluta es indiferente (falta de apetencia -das Potenzlose), porque en cada producto está ya actuando la no diferencia, y además, porque aquella unidad absoluta significaría el fin de la actividad y la libertad (el fin de todo poder), por eso aquel ser incondicional es entendido como puro inicio o puro poder, o mejor anfangen könnend. Schelling piensa el absoluto como una reine Einheit entre el absoluto y su manifestación, de tal modo que éste se puede pensar a través de lo que él produce, o mejor, de lo que es producido desde él, en el sentido de nacido: la Natur in Gott pensada en términos de Hervorgang. Con otras palabras, la meta de la productividad son los productos, aunque la determinabilidad permanezca libre de toda determinación, y una liberación respecto de las formas limitadas se eternice (perpetuitas vitae).

Siendo así, la realidad de Dios como causa del mundo, el ser (considerado fuera de sus determinaciones; en sentido verbal), ni existe fuera de sus manifestaciones, ni puede ser pensado o entendido fuera de ellas. Pero si esto es así, Schelling está señalando un límite a la filosofía allí donde ella misma creía tener su razón de ser: a la hora de pensar la causa de las causas. Este límite no es un límite del entendimiento, no es para Schelling un problema del conocimiento, sino que se trata de una razón ontológica: no hay ningún ser que entender fuera de lo siendo. Retomemos otra vez la pregunta rectora de este comentario: ¿qué hace de Schelling un autor que merezca ser rescatado, estudiado y posiblemente considerado como la cima del idealismo alemán, la obra en la que el idealismo alcanza el punto álgido tras el cual no puede haber ya más idealismo, y en base a qué podemos decir que Schelling inicia la actual situación del quehacer filosófico actual, plural y desvertebrado? Aquí creo que se encuentra la clave: si Schelling habla de una sustancia incondicional que sólo puede ser considerada

9 Cfr., Heidegger, Der Satz von Grund, GA 10.

10 Ibid, 144. 
en sus consecuencias según la fórmula $\mathrm{A} / \mathrm{a}$, entonces tenemos un problema: si ese ser es, como dice Schelling, la realidad de lo siendo, lo que hace ser no puede ser buscado fuera de lo siendo. ¿Estamos entonces ante una suerte de límite de la filosofía tradicional? ¿Del tema de la filosofía, de la arché, no se puede hablar? ¿Mejor es callarse? ¿Acaso cabe elaborar una teoría del conocimiento distinta que pueda dar cuenta de eso ante lo que Schelling retrocede? ¿Hay que volver a realizar la pregunta por el ser a partir de la diferencia ontológica? ¿O es mejor buscar otro modo de hacer filosofía que no sea idealista y que no tenga que enfrentarse al problema que Schelling nos hereda? ¿Qué es filosofía? ¿Cuáles son sus temas? ¿Cuál su método? Debe llamarse la atención sobre el hecho de que ya no se trata sólo de la pregunta empirista por las posibilidades del entendimiento en la línea del empirismo inglés, porque con Schelling la pregunta se vuelve ontológica: ¿cabe concebir un ser fuera de los límites de lo condicionado? El problema de teoría del conocimiento es un problema heredado tras el hallazgo ontológico de este nuevo sentido del ser del que no puede dar cuenta la teoría del conocimiento tradicional y del que ninguna sospecha tenía el empirismo inglés, abanderando Schelling un nuevo empirismo: el empirismo metafísico, que no estudia el ser de las ideas, sino el ser denominado Dios real, ${ }^{11}$ que también puede ser denominado Realität a secas, que es das schlechthin Unbegreifliche y que se distinguiría de las ideas (posibilidades). Pero aunque tuviera razón Schelling ¿por qué debería ser imposible entender este ser real? ¿No deberíamos buscar más bien nuevas categorías para pensar ese nuevo sentido del ser descubierto? ¿Caben ejercicios intelectuales más altos a partir de los cuales superar esta incapacidad del conocimiento ${ }^{12}{ }^{\mathrm{O}} \mathrm{O}$ se trata de una vereda abierta para la reiteración de la pregunta por el ser que habría tenido como pensadores iniciales a los presocráticos Anaximandro y Heráclito? ${ }^{13}$ La polémica está servida, el fin del idealismo gestado, y el campo de batalla para la crisis de la filosofía del siglo siguiente preparado.

Por si acaso este breve comentario hubiera servido para que en alguien brotara esa curiosidad científica que le impulsara a conocer uno de los grandes sucesos que juegan un papel decisivo en el origen del modo de hacer la filosofía actual que hemos heredado, tan plural y enfrentado, donde unos se acusan a otros de no hacer la filosofía que debe hacerse, dejo a continuación como herramienta para iniciar este camino una suerte de mapa de la bibliografía de Schelling que pueda ser usado a modo de linterna para moverse por ese filosofar que Hegel definió como la noche donde todas las vacas son negras.

11 «namentlich den wirklichen Gott», PhO, SW XIII, 140.

12 Cfr., J.A. García, Principio sin continuación, Málaga: Universidad de Málaga, 1998.

13 Cfr., Heidegger, Parmenides, GA 54. 
Para la selección de esta bibliografía se ha partido de la colección de obras de la Kommission zur Herausgabe der Schriften von Schelling de la Bayerische Akademie der Wissenschaften. Me he atrevido a incluir algún trabajo que he considerado importante y que no he encontrado allí. Se ha añadido también a dicha selección un parágrafo especial para mencionar algunas de las contribuciones científicas más relevantes del grupo de investigación sobre Schelling de la Universidad de Málaga, cuya labor, en la tarea aquí reivindicada, es, desde mi punto de vista, rectora. También he considerado fundamental exponer un registro de las obras de Schelling y de las principales ediciones de la misma, junto con la indicación de algunas de las bibliografías sobre Schelling más actuales.

\section{EdICIONES DE LAS OBRAS DE SCHELLING}

AA F.W.J. Schelling: Historisch-kritische Ausgabe. Edita la SchellingKommission de la Bayerische Akademie der Wissenschaften. Los editores son: H.M. Baumgartner, W.G. Jacobs, H. Krings y H. Zeltner.

SW F.W.J. von Schellings sämmtliche Werke. Es la referencia habitual. Se trata de la edición de su hijo: Karl F. August Schelling. En Stuttgart/Augsburg, durante los años 1856-61. [= Schellings Werke, a partir de 1927. Reordenación de M. Schröter que sigue a la edición original, y que mantiene la indicación de la paginación de la sämmtliche Werke]

SW CD F.W.J. von Schelling‘s sämmtliche Werke (1856-1861). CD-ROM/ Windows-Version. Edita: E. Hahn. Berlin, 1998.

\section{BibliografíAs SOBRE SCHELLING}

Se puede consultar la bibliografía en la web de la Schelling-Kommission der Bayerischen Akademie der Wissenschaften:

www.schelling.badw.de/datenbanken/index.html.

Dos muy famosas, pero ya antiguas son la de Schneeberger en Bern: Frankche 1954, y la de Sandkühler en Stuttgart: Metzler 1970.

Más actuales son las siguientes:

La de Sebastian Schwenzfeuer en 2008, atendiendo especialmente a la relación entre Heidegger y Schelling. Se puede descargar desde esta dirección:

http://www.schelling-gesellschaft.de/mat/Bibliographie_Schelling_Heidegger.pdf

Una muy interesante, en 2004, se debe a Andreas Jürgens, que contó con la colaboración de Hans Jörg Sandkühler. Se puede consultar aquí: http://www. philosophie.uni-bremen.de/uploads/media/Schelling-Bibliographie-2004.pdf 
IV. Registro de ObRas de SCHELLING POR ORDEN CRONOLÓGICO ${ }^{14}$

1790

Die Ursprache des Menschengeschlechte.

1792

Die Möglichkeit einer Philosophie ohne Beinamen, nebst einigen Bemerkungen über die Reinholdische Elementarphilosophie

Die Übereinstimmung der Kritik der teoretischen und praktischen Vernunft, besonders in Bezug auf den Gebrauch der Kategorien und der Realisierung der Idee einer intelligibelen Welt durch ein Factum in der Letzteren

Antiquisimi de prima malorum humanorum origine philosophematis Genesis III explicandi tentamen criticum et philosophicum.

1793

Problem eines Kommentars über die früheste Geschichte Iesus nach Lukas und Matthäus

Verschiedene Vorstellungsarten Pauli und Philosophemen der alten Welt 1794

Über die Möglichkeit einer Form der Philosophie überhaupt.

1795

De Marcione Paullinarum epistolarum emendatore.

Vom Ich als Prinzip der Philosophie oder über das Unbedingte im menschlichen Wissen.

\section{6}

Neue Deduktion des Naturrechts.

Aeltestes Systemprogramm des deutschen Idealismus. 1796-97

Abhandlungen zur Erläuterung des Idealismus der Wissenschaftslehre. 1797

Einleitung zu den Ideen zur einer Philosophie der Natur, als Einleitung in das Studium dieser Wissenschaft.

Ideen zu einer Philosophie der Natur.

14 Para la redacción de este apartado se ha tomado por base el registro realizado por García, J.A. «Cronología Biográfica de Schelling» en Schelling F.W.J., Lecciones muniquesas para la historia de la filosofía moderna, Málaga: Edinford, 14-18. Existe también un registro confeccionado por Leyte-Cortés «Registro de obras de Schelling» en: Investigaciones filosóficas sobre la esencia de la libertad humana y los objetos con ella relacionados, Barcelona: Anthropos, 77-81. 
1797-98

Aus der Allgemeine Übersicht der neuesten philosophischen Literatur.

1798

Von der Weltseele, eine Hypothese der höheren Physik zur Erklärung des allgemeinen Organismus.

1799

Erster Entwurf eines Systems der Naturphilosohpie.

Einleitung zu dem Entwurf eines System der Naturphilosophie, oder über den Begriff der spakulativen Phisik.

1800

Über die Jenaische Allgemeine Literaturzeitung.

Anhang zu dem vorstehenden Aufsatz, betreffend zwei naturphilosophischen recensionen und die Jenasche Literaturzeitung.

Allgemeine Deduktion des dynamischen Prozesses oder der Kategorien der Physik.

Miszellen aus der Zeitschrift für spekulative Physik

System des transzendentalen idealismus.

1801

Anhang zu dem Aufsatz des Herrn Eschenmayer betreffend den wahren Begriff der Naturphilosophie und die richtige Art ihre Probleme aufzulösen.

Über den wahren Begriff der Naturphilosophie.

Darstellung meines Systems der Philosophie.

1802

Bruno, oder über das göttliche und natürliche Prinzip der Dinge.

Über das Wesen der philosophischen Kritik überhaupt, und ihr Verhältnis zum gegenwärtigen Zustand der Philosophie insbesondere.

Über das Verhältnis der naturphilosophie zur Philosophie überhaupt.

Über das absolute Identitässystem, und sein Verhältnis zu dem neuesten Dualismus.

Vorlesung über die Methode des akademischen Studiums.

Tratados y recensiones en el Kritischen Journal der Philosophie.

1802-03

Philosophie der Kunst.

1803

Die vier edlen Metalle.

Über die Konstruktion in der Philosophie.

Über Dante in philosophischer Beziehung. 
Fernere Darstellung aus dem System der Philosophie.

1804

Immanuel Kant.

Philosophie und Religion.

Propädeutik der Philosophie (Würzburger Vorlesungen).

System der gesamten Philosophie und der Naturphilosophie insbesondere.

1805

Vorrede zu den Jahrbüchern der Medizin als Wissenschaft.

Aphorismen zur Einleitung in die Naturphilosophie.

Kritische Fragmente.

Vorläufige Bezeichnung des Standpunktes der Medizin nach Grundsätzen der Naturphilosophie.

Abhandlungen über das Verhältnisses des Reales und Idealen in der Natur.

1806

Darlegung des wahren Verhältnisses der Naturphilosophie zu der verbesserten Fichteschen Lehre.

1807

Über das Verhältnis der bildenden Künste zu der Natur.

1807-09

Artículos y recensiones en los Jenaer und Erlanger Literaturzeitung.

1809

Philosophische Untersuchungen über das Wesen der menschlichen Freiheit und die damit zusammenhängenden Gegenstände.

1810

Clara oder über den Zusammenhang der Natur mit der Geisterwelt.

Stuttgarter Privatvorlesungen.

1811

Über das Wesen deutschlischer Wissenschaft.

Otros trabajos breves.

Primera redacción de las Weltalter.

1812

Denkmal der Schrift von den göttlichen Dingen des Herrn Jacobi, und der ihn in derselben gemachten Beschuldigung eines absichtlich täuschende, Lüge redenden Atheismus. 
Carta a Eschenmayer.

Segunda versión de las Weltalter.

1815

Anmerkungen zu den Gottheiten von Samotrake.

Tercera versión de las Weltalter.

Kunstgeschichtliche Anmerkungen zu Johann Martin Wagners.

1818

Spicilegium observatorium in novissimam Arnobii editionem.

1820

Tratados filológicos y mitológicos.

1821

Erlanger Vorträge, donde están los Initia.

1827

Erste Vorlesung in München.

Zur Geschichte der neueren Philosophie.

1827-41

Reden in den öffentlichen Sitzungen der Akademie der Wissenschaft in München.

1830

Darstellung des philosophischen Empirismus.

1832

Grundlegung der positiven Philosophie.

1833

Über die Bedeutung eines neuentdeckten Wandgemäldes von Pompeji.

1834

Vorrede zu einer philosophischen Schrift Victor Cousins.

1938

Worte zum Andenken des Freiherrn von Moll und Sylvestre Sacys.

1840

Antropologisches Schema.

1841

Andere Deduktion der Prinzipien der positiven Philosophie.

1841-43

Philosophie der Offenbarung.

$1842-52$

Philosophie der Mythologie. 


\section{3}

Die endliche offenbar gewordene positive Philosophie der Offenbarung oder die Enstehungsgeschichte, wörtlicher Text, Beurtheilung und Berichtigung der von Schellingschen Entdeckung über Philosophie überhaupt, Mythologie und Offenbarung des dogmatischen Vristentums im Berliner Wintersemestercursus 1841-42.

Der allgemeinen Prüfung vorgelegt von Paulus.

\section{4}

Darstellung der Naturprozesses.

\section{5}

Vorwort zu H. Steffes nachgelassenen Schriften.

1847

Darstellung der reinrationalen Philosophie.

1850

Abhandlugen über die Quelle der ewigen Wahrheiten.

Vorbemerkungen zu der Frage über den Ursprung der Sprache.

1856 ss

Sämmtilche Werke.

V. Selección DE obras Sobre Schelling DEl GRUPO DE INVESTIGACión DE LA Universidad de MÁlaga ${ }^{15}$

FALGUERAS, I. 1983: «Del saber absoluto a la perplejidad. La evolución de la doctrina de las potencias en Schelling», en: Revista de Filosofía 2, 6, 21-34.

1986: «Libertad y verdad. Esbozo de la cuestión al hilo del pensamiento de Schelling», Anuario Filosófico, 19, 2, 25-60.

1988: «La noción de sistema en Schelling», en: FALGUERAS, I. (Ed.): Los comienzos filosóficos de Schelling. Málaga: Universidad de Málaga, 31-66.

1988: Los comienzos filosóficos de Schelling. Málaga: Universidad de Málaga. 1993: «Estudio introductorio», en Schelling F.W.J., Lecciones muniquesas para la historia de la filosofía moderna, Málaga: Edinford, 21-100.

1999: «El inicio del método de las potencias en Schelling», en: LEYTE, A.

(Ed.): Una mirada a la filosofía de Schelling, Actas del Congreso Internacional

15 Sin duda una de las labores más interesante del grupo de investigación andaluz han sido las traducciones de las obras de Schelling. Las edades del mundo, las lecciones muniquesas o la correspondencia completa con Hegel y Fichte son ejemplo de este quehacer del grupo. Sin embargo, en este informe bibliográfico no se recoge información sobre las traducciones de las obras de Schelling, por lo que no se hará mención tampoco de éstas recién mencionadas. Para los interesados, se puede encontrar en el siguiente enlace la información más actualizada y completa sobre el grupo y sus publicaciones: http://juan.leonardopolo.net/schelling.htm. 
«Transiciones y pasajes: naturaleza e historia en Schelling». Vigo: Universidad de Vigo, 245-256.

— 2001: «Le problème du passage chez Schelling», en : ROUX-VETO (Ed.): Schelling et l'élan du système de l'idéalisme transcendantal. París: Ed. Harmattan, 231264.

GARCÍA, J. A. 1986: Simposio internacional «Los comienzos filosóficos de Schelling», en Pensamiento LXII, 382-4.

— 1990: Comunidad y diferencia entre Hegel y Schelling, en: VV. AA. «Filosofía y cultura a finales del s. XX». Málaga: Ayto. Mijas, 211-7.

— 1990: «Interés actual por Schelling». Philosophica 13, 261-7.

1993: «Cronología Biográfica de Schelling», en Schelling F.W.J., Lecciones muniquesas para la historia de la filosofía moderna, Málaga: Edinford, 14-18.

— 2011: «Heidegger sobre Schelling. La explicación del tiempo y la existencia», en: ROJAS, A.: Lecturas de las lecciones privadas de Stuttgart de Schelling (1810). Málaga: Universidad, 15-24.

GUTIERREZ, R. 1990: Schelling: apuntes biográficos. Málaga: Edinford.

ROJAS, A. 2010: «La influencia de Schelling en la cátedra de historia de la filosofía moderna y contemporánea de la universidad de Málaga». En GARCIA-PADIAL (coords.): Autotrascendimiento. Sevilla: Universidad de Málaga, 301-12.

_ 2011: Lectura de las lecciones privadas de Stuttgart de Schelling (1810), Málaga: Universidad de Málaga.

OCHOA, H. 1990: «La naturaleza desde los conceptos de filosofía positiva y negativa en Schelling», en: Philosophica 13, 241-6.

— 1995: «Filosofía de la naturaleza en Schelling», en: Cuadernos de Filosofía 13, 13-26.

-2005: «O dilema de Schelling», en: ALVES-REY (Ed.), As filosofías de Schelling. UFMG, Belo Horizonte, 93-112.

_ 2006: «El alma del mundo en Schelling», en: Hypnos, 16, 17-31.

VI. BIBLIOGRAFÍA GENERAL SELECCIONADA SOBRE SCHELLING

ANIZ, C. 1973: «Filosofía de liberación humana. Una lección de Schelling», Estudios Filosóficos 22, 425-453.

ANSALDI, S. 1993 : La tentative schellingienne: un systeme de la liberte est-il possible?, Paris: Ed. L'Harmattan.

ASMUTH-DENKER-VATER (Eds.) 2000: Schelling. Zwischen Fichte und Hegel, Amsterdam, Philadelphia: B. R. Grüner.

BAGGESEN, J. 1858: Jens Baggesen's Philosophischer Nachlaß, Bern: Stämpfische Druckerei.

BALTHASAR, H. U. 1947: Prometheus. Studien zur Geschichte des deutschen Idealismus, Heidelberg: F. H. Kerle Verlag.

BAUMGARTNER, H.M. 1975: Schelling. Einführung in seine Philosophie, Freiburg/ München: Alber. 
(Ed.) 1979: Prinzip Freiheit. Eine Auseinandersetzung um Chancen und Grenzen transzendentalphilosophischen Denkens. Freiburg/München: Alber, 171-186.

BAUMGARTNER-JACOBS (Eds) 1996: Schellings Weg zur Freiheitsschrift, Stuttgart: frommann-holzboog.

BAUMGARTNER, H. M. y KORTEN,H., 1996: Schelling, München: Beck.

BEACH E. A., 1948: The Potencies of God(s), New York: State University of New York Press.

BIEDERMANN, C. 1842: Die deutsche Philosophie von Kant bis auf unsere Zeit, ihre wissenschaftliche Entwicklung und ihre Stellung zu den politischen und socialen Verhältnissen der Gegenwart. Zweiter Band, Leipzig: Verlag von Mayer und Wigand.

BOCKSHAMMER, G. F. 1821: Die Freyheit des menschlichen Willens, Stuttgart: J. B. Metzler.

BRACKEN, J. A. 1972: Freiheit und Kausalität bei Schelling, Freiburg i. Br., München: Alber.

BRAECKMAN, A., 1988: «Autonomie, moraliteit en menselijke vrijheid. Over de natuurfilosofische grondslagen van Schellings vrijheidsbegrip in het Freiheitsschrift», en: Bijdragen. Tijdschrift voor filosofie en theologie 49, 277298.

_ 1988: «Voorbij de grenzen van de zuivere rede. De late Schelling over vrijheid als project van de filosofie», en: Tijdschrift voor Filosofie, 50, 422-451.

BRUNEDER, G., 1958: «Das Wesen der menschlichen Freiheit bei Schelling und sein ideengeschichtlicher Zusammenhang mit Jakob Böhmes Lehre vom Ungrund», en: Archiv für Philosophie 8, 101-115.

BUCHHEIM, T. 1992: Eins von Allem, Die Selbstbescheidung des Idealismus in Schellings Spätphilosophie, Meiner, Hamburg.

— 2006: «...eine sehr reele Unterscheidung. Zur Differenz der Freiheitsschrift», ENDERS, MARKUS, UHDE, BERNHARD (Eds.), Scientia \& Religio, FreiburgMünchen: Alber, 182-199.

BUCHHEIM-HERMANNI(Ed.), Alle Persönlichkeit ruht auf einem dunklen Grunde, Berlin: Akademie Verlag.

CLAYTON, P. 1996: Das Gottesproblem, Paderborn: Schöningh.

CRUZ, J., 1993: Ontología de la razón en el último Schelling. Acerca de la introducción a la filosofía de la revelación, Pamplona: Universidad de Navarra.

DIOSDADO, C. 1997: Más allá de la teoría, Sevilla: Kronos.

DUQUE, F., 1998: Historia de la Filosofía Moderna. La era crítica, Madrid: Akal. 1998: «La puesta en libertad de la filosofía. El concepto de libertad y la libertad del concepto en Schelling», in: Daimon 16, 41-56.

- 1999: «Die Philosophie in Freiheit setzen. Freiheitsbegriff und Freiheit des Begriffs bei Schelling», en: Fichte-Studien 15, 169-188.

EHRHARDT, W. 1982: «F. W. J. Schelling: Die Wirklichkeit der Freiheit», en: SPECK, J. (Eds), Grundprobleme der großen Philosophen, Göttingen: Vandenhoeck \& Ruprecht, II. 109-144. 
ESPOSITO, C. 1988: Libertà dell'uomo e necessità dell'essere. Heidegger interpreta Schelling, Bari: Ecumenica Editrice.

FÉHER-JACOBS 1999: Zeit und Freiheit, Budapest: Kétef.

FRAN, M., 1985: Eine Einführung in Schellings Philosophie, Frankfurt: Suhrkamp.

FRANK, M., y KURZ, G., (Eds) 1975: Materialien zu Schelling philosophischen Anfängen, Frankfurt: Suhrkamp.

FRIEDERICH, H.J. 2009: Der Ungrund der Freiheit im Denken von Böhme, Schelling und Heidegger, Stutgart: frommann-holzboog.

FUHRMANS, H., 1940: Schellings letzte Philosophie. Die negative und die positive Philosophie im Ein satz des Spätidealismus, Berlin: Junker und Dünnhaupt.

FUJITA, M. 2000: «Hegels Phänomenologie des Geistes und Schellings Freiheitsschrift», en: MATSUYAMA-SANDKÜHLER (Eds), Natur, Kunst und Geschichte der Freiheit, Frankfurt a.M.: Peter Lang, 115-126.

GROOS, F. 1819: Die Schellingische Gottes- und Freiheitslehre vor den Richterstuhl der gesunden Vernunft vorgefordert. Die Vierzehnte der Betrachtungen über moralische Freiheit, Unsterblichkeit der Seele und Gott, Tübingen: Heinrich Laupp.

GUEROULT, M. 1954 : «La philosophie Schellingienne de la liberté», en: Studia Philosophica, XIV, 146-161.

HABERMAS, J. 1954: Das Absolute und die Geschichte. Von der Zwiespältigkeit in Schellings Denken. Diss, Bonn: H. Bouvier u. Co. Verlag.

HARTMANN, N. 1923: Die Philosophie des deutschen Idealismus, Berlin, New York: Walter de Gruyter.

HEIDEGGER, M. 1971: Über das Wesen der menschlichen Freiheit (1809), Tübingen: Niemayer.

1971, «Ausgewählte Stucke aus dem Manuskripten zur Vorbereitung des SchellingSeminars S.S. 1941», en: Feick, H. (Ed..), Schelling Abhandlung über das Wesen der menschlichen Freiheit (1809), Tübingen: Niemayer.

— 1982: Vom Wesen der menschlichen Freiheit. Einleitung in die Philosophie, Frankfurt am Main: Klostermann.

1991: Die Metaphysik des deutschen Idealismus. Zur erneuten Auslegung von Schelling: Philosophische Untersuchungen über das Wesen der menschlichen Freiheit und die damit zusammenhängenden Gegenstände (1809), Frankfurt am Main: Klostermann.

HEIMSOETH, H. 1958: Die Sechs großen Themen der Abendländischen Metaphysik, Darmstadt: Kohlhammer Verlag.

HEINE, F. 1980: Freiheit und Totalität. Zum Verhältnis von Philosophie und Wirklichkeit bei Fichte und Hegel, Bonn: Bouvier.

HENNIGFELD, J. 2001: Friederich Wilhelm Joseph Schellings >Philosophische Untersuchungen über das Wesen der menschlichen Freiheit und die damit zusammenhängenden Gegenstande, Darmstadt: Wissenschaftliche Buchgesellschaft.

HENNIGFELD-STEWART 2003: Kierkeggard und Schellin, Berlin-Newyork: Gruyter. 
HERMANNI, F. 1994: Die letzte Entlastung, Wien: Passagen Verlag.

HEUSER-KLEBER, L. M., 1986: Die Produktivität der Natur. Schellings Naturphilosophie und das neue Paradigma der Selbstorganisation in den Naturwissenschaften, Berlin: Dunker und Humboldt.

HÖFFE O., PIPPER A.,(Eds) 1995: F. W.J. Schelling über das Wesen der menschlichen Freiheit, Berlin: Akad Verlag.

HOGREBE, W. 1989: Prädikation und Genesis, Berlin: Suhrkamp.

HÜHN-JATZEN (Eds.), 2010: Heideggers Schelling-Seminar (1927/28), Stuttgart-Bad Canstatt: frommannn-holzboog.

HUTTEL, A., 1996: Geschichtliche Vernunft, Frankfurt a.M.: Suhrkamp.

IRLENBORN, B. 2000: «Das Gute ist das Böse», en: Archiv für Begriffsgeschichte 42, 1 (2000), 155-178.

JACOBS, W.G 1999: «Das Weltbild der modernen Naturwissenschaften und die Schellingsche Naturphilosophie», en: FEHÉR-JACOBS (Eds.), Zeit und Freiheit, Budapest: Kétef, 37-54.

- 2001: «Anerkennung und Organismus», en: HOFMANN-THURNHERR, (Eds.), Anerkennung, Freiburg / München: Karl Alber, 2001.

- 2004: «Friedrich Wilhelm Joseph (von) Schelling. Vernunft und Wirklichkeit», en: FLEISCHER-HENNINGFELD, (Eds.), Philosophen des 19. Jahrhunderts. Darmstadt: Wissenschaftliche Buchgesellschaft und Primus Verlag, 2, 55-69. 2004: Schelling lesen. Stuttgart-Bad Cannstatt: Wallstein.

2005: «Schelling -Denker der Freiheit- Philosophie und Leben», en: zur debatte. Themen der Katholischen Akademie in Bayern, 35, 2. München, 26-27.

- 2006: «Fichtes Wissenschaftslehre in Schellings Spätphilosophie», en: DIETZSCH-FRIGO (Eds.) Vernunft und Glauben. Ein philosophischer Dialog der Moderne mit dem Christentum. Père Xavier Tilliette SJ zum 85. Geburtstag. Berlin: Vernunft und Glauben, 191-201.

-2006: «Freiheit und Anfang im Denken Schellings», en: EDITH-KLAUS (Eds.), Hans Dieter Klein Geist und Willensfreiheit, Würzburg: Klassische Theorien von der Antike bis zur Moderne, 171-184.

- 2007: «Differenz und Freiheit. Zum metaphysischen und kritischen Denken im Deutschen Idealismus», en: WESTERKAMP-LÜHE (Eds.) Metaphysik und Moderne. Ortsbestimmungen philosophischer Gegenwart. Würzburg: FS Claus-Artur Scheier, 41-53.

-2011: «Natur in der Kunst», en: DANZ-JANTZEN (Eds.), Gott, Natur, Kunst und Geschichte, Göttingen: Vienna University Press, 85-102.

JÜRGENSEN, S. 1997: Freiheit in den Systemen Hegels und Schellings, Würzburg: Königshausen \& Neumann.

KILE, F. O. 1965: Die theologischen Grundlagen von Schellings Philosophie der Freiheit, Leiden, Köln: E. J. Brill.

KNATZ, L. 1999: Geschichte - Kunst-Mythos : Schellings Philosophie und die Perspektive einer philosophischen Mythostheorie, Würzburg: Königshausen \& Neumann, 1999. 
KOELLER, D. W. 1992: The physics of freedom: the beginnings of Schelling's philosophy of nature, Ann Arbor, Mich: UMI.

KOLOSWKI, P 2001: Philosophie der Offenbarung, Paderborn: Schöningh.

KORSCH, D. 1980: Der Grund der Freiheit, München: Kaiser.

KRINGS, H. 1985: «Natur als Subjekt. Ein Grundzug der spekulativen Ohysik Schellings», en: HECKMANN-KRINGS-MEYER (Eds.), Referate, Voten und Protokolle der II. Internationalen Schelling-Tagung Zürich 1983, Stuttgart-Bad Cannstatt: Meyer, 111-128.

_ 1994, «Genesis und Materie -Zur Bedeutung der Timaeus-Handschrift für Schellings Naturphilosophie», en: Schelling F.W.J, Timaeus (1794), Stuttgart-Bad Canstatt: Frommann-Holzbog.

KRÜGER, M. D., 2008: Göttliche Freiheit: die Trinitätslehre in Schellings Spätphilosophie, Tübinge: Mohr Siebeck.

LAUTH, R. 1994: «Kann Schellings Philosophie von 1804 als System bestehen? Fichtes Kritik», in: Kant-Studien 85, 1, 48-77.

LEYTE, A. (Ed) 1999: Una mirada a la filosofía de Schelling, Vigo: Universidad de Vigo.

— 1996: «Schelling y la música», Anuario filosófico 29, 54, 107-124.

1998: Las épocas de Schelling, Madrid: Akal.

_ 1999: «La materia y la idea: en torno a la filosofía de la naturaleza de Schelling», Laguna 6, 71-98.

2000: «De Húle a Materie: de Aristóteles a Schelling», Daimon 21, 87-96.

_ 2009: «De la universidad absoluta en Schelling a la universidad existencial en Heidegger: ¿una continuidad?», en ONCINA, F. (coords.), filosofía para la universidad, filosofía contra la universidad: de Kant a Nietzsche, Madrid: Dykinson.

LOOCK, R. 2007: Schwebende Einbildungskraft. Konzeption theoretischer Freiheit in der Philosophie Kants, Fichtes und Schellings, Würzburg: Königshauesen und Neumann.

MAUGÉ,F. 1901 : «La liberté dans l'idéalisme transcendantal de Schelling», en: Archiv für Geschichte der Philosophie 14, 361-383.

MARQUET, J-F. 1973 : Liberté et existence. Étude sur la formation de la philosophie de Schelling, Paris: Édition Gallimard.

MAX, W. 1997: Schelling: Geschichte, System, Freiheit, Freiburg-München: Alber

MENDE, E. 1978: «Die Aktualität des Freiheitsproblems und Schelling», en: Philosophischer Literaturanzeiger 31, 1, 198-206.

MICHAELIS, C. F. 1974: Über die Freiheit des menschlischen Willens, Leipzig: Leipzig.

MILLUCCI, M., 1996: «La Freiheitsschrift del 1809 come momento decisivo tra la filosofia dell'identità e il rilievo dell'esistenza nel pensiero di Schelling», en: Rivista di Filosofia Neo-Scolastica 88, 2, 205-222.

MOISO, F. 1990: Vita natura libertà. Schelling (1795-1809), Milano: Mursia.

MOKROSCH, R. 1976: Theologische Freiheitsphilosophie, Frankfurt a. M.: Klostermann. 
NAMENLOS 1821: Über das Wesen der menschlichen Freiheit. Zur Erläuterung und Würdigung der Schelling'schen Theorie, diese Lehre betreffend, Leipzig: Voss.

OLIVER, R. L. 1977: Schelling and Kierkegaard: Experimentations in Moral Autonomy. Diss, Ann Arbor (Mich.): Univ. Microfilms Internat.

PEETZ, S. 2006: «Der Grund der Freiheit», en: Der blaue Reiter, Journal für Philosophie, 2, 55-59.

POREBA, M. 1993: «Zum Freiheitsproblem im frühesten systematischen Denken Schellings», en: Nowa krytyka, 4, 7-23.

SANDKÜHLER, H. 1968: Freiheit und Wirklichkeit: zur Dialektik von Politik und Philosophie bei Schelling, Frankfurt am Main: Suhrkamp.

(Ed.) 2000: Natur, Kunst und Geschichte der Freiheit, Frankfurt a.M.: Peter Lang, 93-114.

1970: F. W. J. Schelling, Stuttgart: Metzler.

SCHEIER, C.A., 1996: "Kants dritte Antinomie und die Genese des tragischen Gedankens: Schelling 1795-1809», en: Philosophisches Jahrbuch, 103, 1, 76-89.

SCHULZ, W. 1955: Die Vollendung des deutschen Idealismus in der Spätphilosophie Schelling, Stuttgart: Kohlhamer.

SCHURR, A., 1974: Philosophie als System bei Fichte, Schelling und Hegel, StuttgartBad Cannstatt: Frommann-Holzboog.

SERRANO, V. 2008: Absoluto y conciencia. Una introducción a Schelling, Madrid: Plaza y Valdés.

SHIKAYA, T., 2000: «Von der Identität zur Individuation», en: MATSUYAMA-

STURMA, D. 1994: «Zur Wiedererwägung eines Begriffs positiver Freiheit. Das Schelling-Symposion in Kampen», en: Zeitschrift für philosophische Forschung, 48, 2, 284-491.

SWARZ, J. 1935: «Die Lehre von den Potenzen», en: Kant-Studien, 40, 118-148.

THEUNISSEN, M. 1965: «Schellings anthropologischer Ansatz», en: Archiv für Geschichte der Philosophie, 47, 2, 174-189.

TILLIETE, X., 1987 : L'Absolu et la philosophie, Paris: PUF.

- 1970: Schelling. Une philosophie en devenir, t. I, Le Système vivant, 1794-1821, t. II, La Dernière Philosophie, 1821-1854, Paris: Vrin.

VERGAUWEN, G. 1975: Absolute und endliche Freiheit. Schellings Lehre von Schöpfung und Fall, Freiburg/Schweiz: Universitätsverlag.

WATANABE, J. 2000: «Der Mensch in der Entzweiung seines inneren Wesens», en: MATSUYAMA-SANDKÜHLER (Eds.), Natur, Kunst und Geschichte der Freiheit, Frankfurt a.M.: Peter Lang, 127-147.

WHITE, A. 1983: Schelling: An Introduction to the System of Freedom, New Haven (Conn.): Yale University Press.

ZANTWIJK, T. 2000: Pan-Personalismus, Stuttgart: frommann-holzboog.

ZERBST, A. 2011: Schelling und die bildende Kunst zerbst, Fink: Paderborn.

ZIZEK, S. 1997: The Abyss of Freedom / Ages of the World, Ann Arbor: Univ. of Michigan Press 
ALEJANDRO ROJAS JIMÉNEZ es investigador postdoctoral de la Alexander von HumboldtStiftung en la Kommission zur Herausgabe der Schriften von Schelling de la Bayerische Akademie der Wissenschaften y en la Ludwig-Maximilians-Universität von München (Alemania).

Líneas de investigación:

El Idealismo alemán y sus consecuencias actuales

Teorías de la verdad

Filosofía contemporánea

Publicaciones recientes:

(2012) "La influencia de Schelling en la lección de Heidegger de 1942/43”, en Thémata. Revista de Filosofía 45, 383-394 (Universidad de Sevilla)

(2011) "El papel de una <<apotencia>> fundamental en la filosofía tardía de Schelling, y su influencia en la filosofía tardía de Heidegger," en Revista de Filosofía 36/2,109-131 (Universidad Complutense de Madrid)

Correo electrónico: rojasj_a@yahoo.es 
